\title{
Cord complications: associated risk factors and perinatal outcome
}

\author{
Vijayata Sangwan, Smiti Nanda, Mukesh Sangwan, Roopa Malik, Manisha Yadav
}

Department of Obstetric \& Gynecology, Pt. B. D. Sharma PGIMS, Rohtak, India.

Email: vsangwan03@gmail.com

Received 30 July 2011; revised 4 September 2011; accepted 15 September 2011.

\section{ABSTRACT}

Objective: To assess the perinatal outcome in patients with cord presentation and cord prolapse over a period of 2 years and to assess the obstetric risk factors associated with these cord complications. Design: Retrospective case study. Setting: A tertiary health care centre of India. Sample: All cases of cord presentation and cord prolapse diagnosed in the department over a period of 2 years (2009-2010). Methods: The information regarding maternal presentation at the time of admission, mode of delivery, and neonatal outcome was retrieved from case sheets of patients available in Medical Record Department. Maternal factors in terms of age, parity, gestational age, mode of presentation, mode of delivery were analysed. Foetal outcome was assessed by Diagnosis to Delivery Interval, APGAR score at 0 minutes and 5 minutes, foetal weight and need for NICU admission. Results: There were 53 cases of cord complications, out of which 7 $(13.20 \%)$ had cord presentation and $46(86.80 \%)$ had cord prolapse. Among these 46 patients, 11 patients developed cord prolapse in labour room ( 3 after artificial rupture of membranes and 8 after spontaneous rupture of membranes), rest 34 patients presented in emergency with cord prolapse. There were $79.24 \%$ multiparous patients, 16 patients had malpresentations and breech was commonest malpresentation. Forty one patients had positive cord pulsations at the time of admission, all these patients underwent emergency caesarean section and all had live babies. Twelve patients reported to us with absent cord pulsation, 3 among these underwent section for obstructed labour with hand and cord prolapse, rest 9 delivered vaginally. The average DDI was 26.00 minutes, DDI in patients developed cord prolapse in hospital was 18.90 minutes \pm 5.48 minutes, whereas in patients admitted with cord prolapse as emergency, the DDI was 29.34 minutes \pm 6.37 minutes $(p<0.05)$. Conclusion: Analysis of the study concluded that the delive- ry interval between cord prolapse and delivery of the foetus is very important. Shortening of this interval can decrease neonatal complication at birth and reduce NICU admission.

Keywords: Cord Complications; Perinatal; Caessrean Section

\section{INTRODUCTION}

Umbilical cord prolapse is a rare obstetric complication usually necessitating emergent delivery. It is life threatening emergency for the foetus as the blood flow through the umbilical vein is compromised by cord compression. The incidence of cord prolapse reported in literature ranges from $0.1 \%-0.6 \%$ and has remained constant over the last centuary [1]. A number of unavoidable risk factors have been associated with increased incidence of cord prolapse which include malpresentation, prematurity, multiple gestations, premature rupture of membranes \& polyhydramnios. Remarkably high correlation of cord prolapse and obstetric interventions such as foetal scalp electrode application, insertion of intrauterine catheter, induction of labour with artificial rupture of membranes (ARM) is reported. Post natal mortality and morbidity has fallen significantly as a result of advances in management of cord prolapse and NICU care. Diagnosis to Delivery Interval (DDI) of 20 minutes - $30 \mathrm{mi}-$ nutes has been recommended by various international obstetric societies $[2,3]$. The primary objective of this study was to assess the perinatal outcome in patients of cord prolapse and cord presentation over a period of 2 years. A secondary objective was to assess the obstetric risk factors associated with these cord complications.

\section{METHODS}

This was a retrospective study conducted at Pt. B D Sharma PGIMS Rohtak, a tertiary health care unit with an average of 11,000 - 12,000 deliveries per year. The information was retrieved from case sheets of patients 
available in Medical Record Department. Maternal condition at the time of admission in terms of age, parity, gestational age, foetal presentation, status of membranes and mode of delivery were statistically analysed (Independent sample $\mathrm{T}$ test). Different cord complications were defined as follows to made diagnosis. Overt cord prolapse was defined as the descent of the cord through the cervix in to the vagina or onto the vulva. Cord presentation meant the presence of one or more loops of umbilical cord between the fetal presenting part and the fetal membranes overlying the cervical os. At admission, the fetal condition was assessed and those with maturity of $>32$ weeks and positive cord pulsations were immediately shifted for caesarean section. In these patients, in order to alleviate pressure on cord, the cord was immediately placed high up in vagina, presenting part was elevated simultaneously and bladder filled with $500 \mathrm{ml}$ normal saline (Vago method) [4-10] until the patient was on operating table. Foetal outcome was assessed in terms of neonatal weight, APGAR score at 0 minutes and $5 \mathrm{mi}-$ nutes and NICU admission. Neonatal asphyxia was identified by the pediatrician on clinical grounds. In the patients getting admitted in emergency with complaint of leaking per vaginum for varying duration, it was difficult to comment on exact time of cord prolapse. Thus, Diagnosis to Delivery Interval was calculated as the time between reporting of patient and delivery of the foetus.

\section{RESULTS}

Amongst 22,681 deliveries over a period of 2 years, 53 cases of umbilical cord complications were identified, giving the incidence of cord complication as $0.23 \%$. Out of these 53 patients, $18(33.96 \%)$ patients reported to us with intact membranes and $35(66.07 \%)$ patients reported to us with complaints of leaking per vaginum for varying duration and cord prolapse. In 18 patients with intact membranes, 7 had cord presentation, 3 patients developed cord prolapse after artificial rupture of membranes in the labour room and 8 admitted patients developed spontaneous leaking per vaginum leading to cord prolapse. The details regarding patient profile are described in Table 1.

Table 2 explains the risk factors of cord prolapse in our study. Malpresentation was the commonest cause and breech was the commonest malpresentation. The cervical dilatation at the time of presentation was $<5 \mathrm{~cm}$ in 34 $(64.11 \%)$ and $>5 \mathrm{~cm}$ in $19(35.89 \%)$ patients.

Table 3 shows about perinatal characteristics in the study. At admission, cord pulsations were present in 41 patients, rest of the fetuses (12) were already dead. All patients with positive cord pulsations were immediately shifted to operation theatre for urgent LSCS and all had live babies. The fetal maturity in these was 32 weeks (1),
33 weeks - 36 weeks ( 8 ), rest 32 were $>36$ weeks. Among these, birth weight of 18 babies was $1.5 \mathrm{~kg}$ to $2.5 \mathrm{~kg}, 23$ babies were of $2.6 \mathrm{~kg}$ to $3.5 \mathrm{~kg}$, and 2 babies weigh more than $3.6 \mathrm{~kg}$. Apgar score was $<5$ at first minute in 8 neonates, in 5 neonates it remained $<5$ at $10 \mathrm{~min}$ and they were admitted in NICU. These 5 fetuses were born to mothers who had been admitted as emergencies with cord prolapse.

Of the 12 patients admitted with absent cord pulsations, 3 mothers had presented with obstructed labour and had to be taken up for section for the sake of mother. Rest 9 were delivered vaginally.

Table 1. Characteristic of patients with cord complications.

\begin{tabular}{|c|c|c|}
\hline Characteristics & No. of patients & $\%$ of patient \\
\hline \multicolumn{3}{|l|}{ Age group (years) } \\
\hline$<20$ & 03 & 5.6 \\
\hline $21-25$ & 37 & 69.81 \\
\hline$>25$ & 13 & 24.52 \\
\hline \multicolumn{3}{|l|}{ Parity } \\
\hline Nulliparous & 11 & 20.75 \\
\hline multiparous & 42 & 79.24 \\
\hline \multicolumn{3}{|l|}{ Gestational age (weeks) } \\
\hline$<37$ & 09 & 16.98 \\
\hline$>37$ & 44 & 83.01 \\
\hline \multicolumn{3}{|l|}{ Presentation } \\
\hline Vertex & 37 & 69.81 \\
\hline Nonvertex & 16 & 30.18 \\
\hline \multicolumn{3}{|l|}{ Cord complications } \\
\hline Cord presentation & 07 & 13.20 \\
\hline Cord prolapse in hospital & 11 & 20.75 \\
\hline Cord prolapse at admisson & 35 & 67.92 \\
\hline \multicolumn{3}{|l|}{ Cervical dilatation $(\mathrm{cm})$} \\
\hline$<5$ & 34 & 64.15 \\
\hline$>5$ & 19 & 35.84 \\
\hline
\end{tabular}

Table 2. Risk factors for cord prolapse.

\begin{tabular}{cccc}
\hline Sr. no. & Risk factor & No. of patients & \% of patient \\
\hline 1 & Multiparity & 42 & 79.24 \\
& Malpresentation & 16 & 30.18 \\
2 & Breech & 08 & 15.09 \\
& Transverse lie & 04 & 7.54 \\
& Compound presentation & 04 & 7.54 \\
3 & Unengaged head & 09 & 16.98 \\
5 & Twins & 02 & 3.77 \\
6 & Prematurity & 09 & 16.98 \\
\hline
\end{tabular}


Table 3. Perinatal characteristics.

\begin{tabular}{|c|c|c|}
\hline Characteristic & No. of patients & $\%$ of patient \\
\hline \multicolumn{3}{|c|}{ Cord pulsations at diagnosis } \\
\hline Present & 41 & 77.35 \\
\hline Absent & 12 & 22.64 \\
\hline \multicolumn{3}{|l|}{ Fetal Maturity } \\
\hline$<28$ wks & 2 & 3.77 \\
\hline $28 w k s-32$ wks & 2 & 3.77 \\
\hline 33 wks - 36wks & 5 & 9.43 \\
\hline$\geq 37$ wks & 44 & 83.01 \\
\hline \multicolumn{3}{|l|}{ Apgar at $1 \mathrm{~min}}$. \\
\hline$<5$ & 10 & 18.86 \\
\hline$>5$ & 31 & 58.49 \\
\hline \multicolumn{3}{|c|}{ Fetal weight at birth $(\mathrm{kg})$} \\
\hline$<2.5$ & 16 & 30.18 \\
\hline$>2.5$ & 37 & 69.81 \\
\hline \multicolumn{3}{|l|}{ Baby status } \\
\hline Live & 41 & 77.35 \\
\hline dead & 12 & 22.64 \\
\hline \multicolumn{3}{|l|}{ Sex } \\
\hline Male & 35 (2 twins) & 66.03 \\
\hline female & 20 & 37.73 \\
\hline \multicolumn{3}{|l|}{ Mode of delivery } \\
\hline Emer. LSCS & 44 & 83.01 \\
\hline NVD & 09 & 16.98 \\
\hline \multicolumn{3}{|l|}{ Baby admission } \\
\hline NICU & 5 & 9.43 \\
\hline With mother & 36 & 67.92 \\
\hline
\end{tabular}

The perinatal mortality in our study was 0.52 per 1000 births. The average DDI in our study was 26.00 minutes. In the patients who had developed leaking in the hospital, the DDI was 18.90 minutes \pm 5.48 minutes with no NICU admission and good Apgar score post delivery; whereas in patients admitted with cord prolapse as emergency, the DDI was 29.34 minutes \pm 6.37 minutes $(\mathrm{p}<$ $0.05)$.

\section{DISCUSSION}

Cord prolapse and cord presentation are the obstetric emergencies that change the management of a simple pregnancy and necessiate urgent delivery. They are associated with high perinatal mortality and morbidity. Successful outcome depends on several maternal and foetal factors. Incidence of cord prolapse in our study was 2.3 per 1000 deliveries .Other studies have reported the incidence of $1.5,1.4$ per 1000 deliveries, 2.7 and 2.8 per 1000 deliveries [2,4-6].

Associated risk factors are those that predispose a woman to have incompletely filled pelvis in the presence of ruptured membranes. In the present study, the risk factors for cord presentation and cord prolapse were found in $39(71.07 \%)$ patients. Multiparity is associated with increased risk of cord prolapse. It is accounted by the fact that in multipara, the engagement of presenting part occurs late, and as a result with rupture of membranes, cord slips down in vagina. In our study, $79.24 \%$ patients were multiparous and $8(15.09 \%)$ were $>$ P4 . Malpresentations were present in $16(30.18 \%)$ and breech was commonest malpresentation. Prematurity was a cause in 9 (16.98\%) patients. Other studies on risk factors associated with cord prolapse have also reported the same factors $[7,8]$

The perinatal mortility in our study was 0.52 per 1000 births. All these deaths were unavoidable as all had absent cord pulsations at the time of presentation. A few studies reported high perinatal mortality [2,9]. Literature suggests that cord prolapse occurring outside the hospital is consistently associated with a high perinatal mortality rate $(38 \%-42 \%)$. In contrast, cord prolapse in a labour room where patient is under monitoring, the perinatal mortality is very low $(0 \%-3 \%)$.

The cause of fetal distress in cord prolapse is cord compression and urgent caesarean section releives that compression. Studies revealed that DDI $>30$ minutes associated with lower Apgar score [1]. In our study DDI was found 26.00 minutes which is well within the recommended range and comparable with other studies reported on cord prolapse. According to international bodies, the time required to perform an emergency cesarean section should not exceed 30 minutes [4]. The ACOG also recommends incision time of 20 minutes - $30 \mathrm{minu}-$ tes to appropriate emergency caessrean section in patient of cord prolapse. Literature suggests that DDI alone is not the only contributing factor [9-12]. The other factors like degree of cord compression, length of cord prolapsed, prior hypoxia, CTG abnormalties may influence foetal outcome. Inspite of widespread prevalence of risk factors of cord prolapse, this problem is very rare. It may be attributed to tone of the cord, its collagenous contents, and its relation to lower segment. Studies also suggest that intrapartum USG in patients prone for developing cord prolapse before ARM may decrease the incidence of cord prolapse $[2,4]$.

The perinatal mortality and morbidity due to cord prolapse has significantly improved over past century as a result of advances in labour management, improved surgical techniques and neonatal intensive care. However, studies have revealed that perinatal outcome can further be improved by a multiprofessional obstetric emergency training sessions [3]. In our study, patients who had developed cord prolapsed in the labor room had average DDI of $18.90 \mathrm{~min}$, and no NICU admission. While pa- 
tients, who presented with cord prolapse, had an average DDI of $29.34 \mathrm{~min}$. This may be because of the extra time, required in preparing patient for operation theatre. The literature suggests that the main reason, why units fail to comply with the 30 minutes target, delay in shifting patient to theatre [3]. Thus in order to achieve the advocated DDI of $<30$ minutes, planned protocols for the management of cord prolapse and the necessary drill of the routine staff to shift the patient on to OT table at the earliest, needs to be strongly followed in the labour rooms.

\section{CONCLUSIONS}

In our study, we concluded that the delivery interval between cord prolapse and delivery of the foetus is very important. Shortening of this interval can decrease neonatal complication at birth and reduce NICU admission. The preparatory time of patient for operation theatre and her shifting to operation theatre where sister and paramedical staff plays important role, is very crucial for the patient. So, there should be written protocol for the staff, alarming system in labour room to alert the whole team should be there, to decrease DDI and improve neonatal outcome.

\section{REFERENCES}

[1] Monique, G.L. (2006) Umbillical cord prolapse CME review article. Obstetrical and Gynecological Survey, 61, 269-277.

[2] Khan, R.S., Naru, T. and Nizami, F. (2007) Umblical cord prolapse-A review of diagnosis to delivery interval on perinatal and maternal outcome. Journal of Pakistan Medical Association, 57, 487-491.

[3] Siassakos, D., Harafa, Z., Sibanda, T., Fox, R., Donald, F., Winter, C. and Draycott, T. (2009) Retrospective cohort study of diagnosis delivery interval with umbilical cord prolapse: The effect of team training. British Journal of Obstetrics and Gynaecology, 116, 1089-1096.

doi:10.1111/j.1471-0528.2009.02179.x

[4] Al Marzowki, A.M. and El Shobokshi, A.S. (2009) Audit of cord prolapse. Management at King Abdulaziz University Hospital. Journal of King Abdulaziz University, Medical Sciences, 16, 35-42.

[5] Bako, B., Chama, C. and Audu, B.M. (Sep2009) Emergency obstetric care in a Nigerian tertiary hospital: A 20 year review of umbilical cord prolapse. Nigerian Journal of Clinical Practice, 12, 232-236.

[6] Traore, Y., et al. (2006) Frequency of cord prolapse: Etiological factors and foetal prognosis in 47 cases in a hea1th centre. Medicine For Mali, 21, 25-29.

[7] Dilbaz, B., Ozturkoglu, E., Dilbaz, S., Oztuck, N., Sivaslioglu, A.A. and Haberal, A. (2006) Risk factors and perinatal outcome associated with umbilical cord prolapse. Archives of Gynecology and Obstetrics, 274, 104107. doi:10.1007/s00404-006-0142-2

[8] Kahana, B., Sheinar, E., Levy, A. and Mazor, M. (2004) Umbilical cord prolapse and perinatal outcomes. International Journal of Gynecology and Obstetrics, 84, 127132. doi:10.1016/S0020-7292(03)00333-3

[9] Faiz, S.A., Habib, F.A., Sporrong, B.G. and Khalil, N.A. (2003) Results of delivery in umbilical cord prolapse. Saudi Medical Journal, 24, 754-757.

[10] Bord, I., Gemu, O., Anleby, E.Y. and Shenhav, S. (2010) The value of bladder filling in addition to manual elevation of presenting foetal part in cases of cord prolapse. Archives of Gynecology and Obstetrics, 15.

[11] Tan, W.C., Tan, L.K., Tan, H.K. and Tan, A.S. (2003) Audit of crash emergency caesarean section due to cord prolapse in terms of response time and perinatal outcome. Annals, Academy of Medicine, 32, 638-641.

[12] Prabulos, A.M. and Philipson, E.H. (1998) Umbilical cord prolapse. Is the time from diagnosis to delivery critical? Journal of Reproduction Medicine, 43, 129-132. 